\title{
ESDA2012-82197
}

\section{DRAFT: TRAJECTORY GENERATION FOR HIGH SPEED PICK AND PLACE ROBOTS}

\author{
Cornelius J. Barnard \\ Sébastien Briot \\ Stéphane Caro \\ Institut de Recherche en Communications et Cybernétique de Nantes (IRCCyN) \\ UMR CNRS 6597 \\ 1 rue de la Noë, BP 92101, 44321 Nantes Cedex 3 France \\ Emails: CorneliusJBarnard@gmail.com \\ Sebastien.Briot@irccyn.ec-nantes.fr \\ Stephane.Caro@irccyn.ec-nantes.fr
}

\begin{abstract}
Currently parallel robots are finding more and more acceptance in high-speed pick-and-place operations. As for all highspeed mechanisms, vibratory phenomena appear and deteriorate accuracy and dynamic performance at the terminal positions of the pick-and-place path. This paper aims to evaluate the effectiveness of several pick-and-place trajectories in terms of vibration reduction. To address this problem, an elastodynamic model of a five bar mechanism is developed and its behaviour is simulated as it traverses each trajectory. Spectral analysis of the vibrations allows the quality of the vibration reduction to be quantified. The results show that the first and second natural frequencies of the system are excited. It is also shown that long cycle times with smooth motion profiles improve residual error norms, but are outperformed by their discontinuous counterparts when short cycle times are imposed.
\end{abstract}

\section{INTRODUCTION}

Nowadays parallel robots are used more and more in highspeed pick-and-place operations. The drive for higher operational speeds and higher payload-to-weight ratios is shifting their designs to more lightweight architectures [?, ?]. The fastest industrial robot, the Quattro by Adept Technologies Inc. [?], reaches more than $15 \mathrm{~g}$ 's of acceleration, allowing up to 4 standard pick-and-place cycles to be performed per second. How-

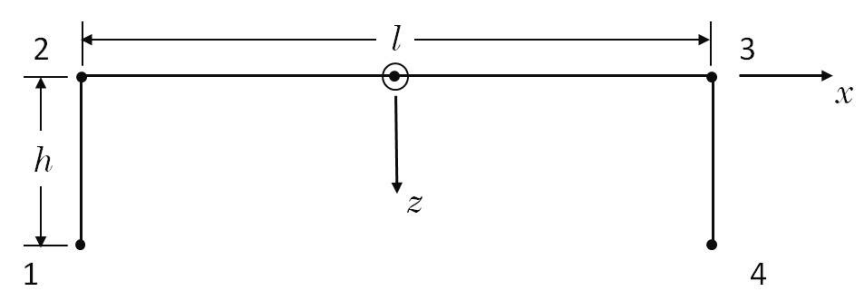

FIGURE 1. Pick-and-place trajectory.

ever, as for all high-speed mechanisms, vibratory phenomena appear that deteriorate accuracy and dynamic performance.

The pick-and-place operation is commonly used by industrial robots involved in both primary handling and case packing $[?, ?]$. The procedure transfers an object from one position to another in a workspace [?]. This standardized geometric path is referred to as the Adept cycle and its most used dimensions are $h=25 \mathrm{~mm}$ and $l=350 \mathrm{~mm}$ [?].

The standard adept cycle, shown in Fig. 1, has square corners that introduce discontinuities in acceleration when traversed. To overcome these discontinuities, extremely high torques must be generated at the actuators, this, coupled with the inertial effect of the moving system, give rise to unwanted vibrations. To avoid these discontinuities the Adept cycle is made smooth by introducing blends at the corners [?,?]. 


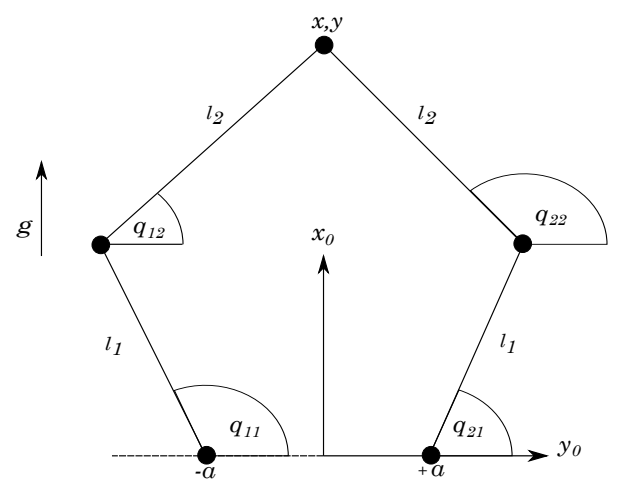

FIGURE 2. Rigid Geometric Model

The rate at which the geometric path is followed is also a source of vibration. The motion profile must be well chosen and designed to minimize excitation of resonances in the structure. The coupling of the geometric path and the motion profile gives rise to the trajectory. Thus, one question arises: what should be the best pick-and-place path for high-speed robots that minimize their vibrations? To the best of our knowledge, this question has not been answered yet.

As a result, this paper aims to define an optimal motion generator based on the Adept typical cycle for the reduction of vibrations in high speed parallel robots performing pick-and-place operations. Vibrations must be reduced by carefully planning the displacements of the end effector. This study is performed on a five-bar mechanism (Fig. 2) [?]. This parallel robot may be seen as the combination of two kinematic chains, each composed of two revolute joints that are linked at the end-effector by a revolute joint. The rigid case assumes that the end effector position $\mathbf{X}$ is known, and hence the joint variables for each leg may be found.

The work firstly develops an elastodynamic model using the method of assumed modes according to the Lagrangian formulation $[?, ?, ?, ?]$. Secondly input trajectories are designed, defining the desired end effector motion along the pick and place path. Lame, clothoid-pair and polar polynomial blends are introduced to smoothen the geometric paths. Seventh degree polynomials, bang-bang and trapezoidal profiles are used for sampling thereby generating the trajectories. Lastly simulations are done in Simulink to excite the five-bar mechanism along the trajectories, vibrational results are obtained and discussed. The simulations are based on industry standards and reveal the vibrational effect of the deformations arising from inertial forces. Spectral analysis of the vibrations show that the first and second natural frequencies of the system are excited. It is shown that long cycle times with smooth motion profiles improve residual error norms, but are outperformed by their discontinuous counterparts when short cycle times are imposed.

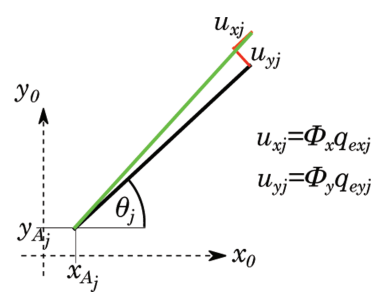

FIGURE 3. Generalized coordinates for each beam, $\mathbf{q}_{\mathbf{e}}=\left[\begin{array}{ll}\mathbf{q}_{\mathbf{e}_{\mathbf{x}}} & \mathbf{q}_{\mathbf{e}_{\mathbf{y}}}\end{array}\right]^{T}$

\section{ELASTODYNAMIC MODELING OF ROBOTS}

Various elastodynamic modeling techniques available to develop the Lagrangian formulation; Finite Element Modeling represents each link as an assembly of a finite number of elements [?]. Linear finite elements make use of polynomial interpolation functions to characterize a link's elastic behaviour [?, ?]. Boundary conditions, changes in geometry and physical properties can be easily accounted for with this method [?]. Lumped Parameter Modeling discretizes links into a series of rigid bodies connected by linear springs introducing the flexible features to the model [?, ?]. Assumed Modes Modeling describes flexible displacements by a truncated modal series, in terms of spatial mode eigen functions and time-varying mode amplitudes [?,?]. The truncated modal series refers to a subgroup of trigonometric functions each depicting the physical modal behaviour of links or beams. This formalism is well-fitted for mechanisms with simple link shapes, such as parallel robots. As a result, this approach will be used for the elastodynamic modeling of the considered five-bar mechanism.

\section{Beam Deflection Modeling}

Let us first define the shape functions, $\Phi$ of the robot links. They are coupled with time varying elastic coordinates $\mathbf{q}_{\mathbf{e}}$ in order to characterize the link deflections $[?, ?]$. In bending, these deflections are perpendicular to the undeformed axial direction of the beam, whereas they are collinear with this axis in tensioncompression deformation (Fig. 3). If $k$ modes of deformations are taken into consideration, the vector $\Phi$ is of size $1 \times k$.

It is assumed that actuated proximal links are rigidly connected to actuators, as such, these links are modeled as beams in bending [?]. For these links, clamped free boundary conditions apply and the $k$-th mode shape function has the following expression:

$$
\begin{aligned}
\phi_{j k}(\xi)= & \sin \left(\alpha_{k} l_{j} \xi\right)+a_{k} \cos \left(\alpha_{k} l_{j} \xi\right) \\
& -\sinh \left(\alpha_{k} l_{j} \xi\right)-a_{k} \cosh \left(\alpha_{k} l_{j} \xi\right)
\end{aligned}
$$

where

$a_{k}$ and $\alpha_{k} l_{j}$ : are modal parameters for mode $k$ $\xi=\frac{x}{l_{j}}: l_{j}$ is length of link $j$ and $x$ is the position along the axial direction of the beam

The first three modal parameters are considered for modeling, the modal parameters are given in Table 1 [?]. Passive distal links 
TABLE 1. Modal parameters for bending deformation

\begin{tabular}{|c||c|c|}
\hline Mode & $a_{k}$ & $\alpha_{k} l_{j}$ \\
\hline \hline 1 & -1.3622 & 1.8751 \\
2 & -0.98186 & 4.6941 \\
3 & -1.0008 & 7.8548 \\
\hline
\end{tabular}

are assumed to be pin-connected, and are thus only loaded in the axial direction. The mode shapes associated with this boundary condition are:

$$
\phi_{j k}(\xi)=\sin \left((2 k-1) \frac{\pi}{2} \xi\right)
$$

where

$$
\xi=\frac{x}{l_{j}}: l_{j} \text { is length of link } j \text { and } x \text { is the position along the axial }
$$
direction of the beam

Having introduced the assumed modes method of modeling and the relevant shape functions, the inertial and stiffness matrices may now be developed.

\section{Lagrangian Formulation}

The Lagrange equations are used to derive the elastodynamic model as it is readily applied to the analysis of closed loop structures. The Lagrangian formulation equates the nonconservative forces acting on the system to the change of energy in the system [?]. The Lagrangian $L$ is defined as the difference between the kinetic energy $E$ and the potential energy $U$ :

$$
L=E-U
$$

The formulation of the Lagrangian terms are shown in the following equations. In these equations, $x_{A}$ and $y_{A}$ denote the position of the beam extremity, $\theta$ the beam orientation and $\mathbf{q}_{\mathbf{e}}$ the set of elastic coordinates representing the deformation of the distal end of the beam (Fig. 3).

\section{Kinetic Energy}

The kinetic energy of a elastic beam, $j$, is expressed using the shape functions $[?, ?, ?]$ :

$$
E=\frac{1}{2} \int_{0}^{l_{j}} \dot{\mathbf{r}}_{\mathbf{M}}^{T} \dot{\mathbf{r}}_{\mathbf{M}} d x
$$

In the above equation, $\dot{\mathbf{r}}_{\mathbf{M}}$ corresponds to the velocity of a point $M$ on the beam and is defined according to the type of deformation modeled on the beam. The position of the point $M$ on the deflected beam can be expressed in the global reference frame as follows (see Fig. 3):

$$
\mathbf{r}_{\mathbf{M}}=\left[\begin{array}{l}
x_{A_{j}} \\
y_{A_{j}}
\end{array}\right]+\mathbf{R}\left(\theta_{j}\right)\left[\begin{array}{c}
l_{j} \xi \\
0
\end{array}\right]+\mathbf{R}\left(\theta_{j}\right)\left[\begin{array}{l}
u_{x_{j}}(\xi, t) \\
u_{y_{j}}(\xi, t)
\end{array}\right]
$$

where

$x_{A_{j}}$ and $y_{A_{j}}$ : Locate the base of link $j$

$\mathbf{R}\left(\theta_{j}\right)$ : Rotation matrix orientating the beam at an angle $\theta_{j}$ $u_{x_{j}}(\xi, t)$ and $u_{y_{j}}(\xi, t)$ : Beam deflections at position $\xi$ and time $t$.

Upon differentiation of Eqn. (5) with respect to time, the velocity of the point $M$, for a beam in pure bending (deformation only being allowed in $u_{y_{j}}(\xi, t)$, i.e. $\left.u_{x_{j}}(\xi, t)=0\right)$ may be found:

$$
\dot{\mathbf{r}}_{\mathbf{M}}=\left[\begin{array}{cccc}
1 & 0 & -\Phi_{y} \mathbf{q}_{\mathbf{e}_{\mathbf{y}}} & 0 \\
0 & 1 & l_{j} \xi & \Phi_{y}
\end{array}\right]\left[\begin{array}{c}
x_{\dot{A}_{j}} \\
y_{\dot{A}_{j}} \\
\dot{\theta}_{j} \\
\dot{\mathbf{q}}_{\mathbf{e}_{\mathbf{y}_{\mathbf{j}}}}
\end{array}\right]
$$

This results in the following mass matrix, dimensioned according to the number of generalized coordinates:

$$
\mathbf{M}_{\mathbf{j}}=m_{j} \int_{0}^{1}\left[\begin{array}{cccc}
1 & 0 & -\Phi_{y} \mathbf{q}_{\mathbf{e}_{\mathbf{y}}} & \mathbf{0} \\
0 & 1 & l_{j} \xi & \Phi_{y} \\
-\mathbf{q}_{\mathbf{e}_{\mathbf{y}_{\mathbf{j}}}{ }^{T}} \Phi_{y}{ }^{T} & l_{j} \xi & \left(l_{j} \xi\right)^{2} & l_{j} \xi \Phi_{y} \\
\mathbf{0} & \Phi_{y}{ }^{T} & l_{j} \xi \Phi_{y}{ }^{T} & \Phi_{y}{ }^{T} \Phi_{y}
\end{array}\right] d \xi
$$

Similarly, the inertia matrix of a beam in tension and compression may be found. Here, deformation is only allowed in $u_{x_{j}}(\xi, t)$ and so $u_{y_{j}}(\xi, t)=0$ :

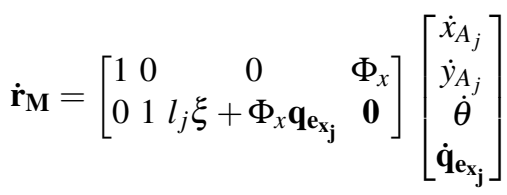

Resulting in the following inertia matrix:

$$
\mathbf{M}_{\mathbf{j}}=m_{j} \int_{0}^{1}\left[\begin{array}{cccc}
1 & 0 & 0 & \Phi_{x} \\
0 & 1 & l_{j} \xi+\Phi_{x} \mathbf{q}_{\mathbf{e}_{\mathbf{j}}} & \mathbf{0} \\
0 & l_{j} \xi+\mathbf{q}_{\mathbf{e}_{\mathbf{x}_{\mathbf{j}}}{ }^{T}} \Phi_{x}{ }^{T} & \Delta & \mathbf{0} \\
\Phi_{x}{ }^{T} & \mathbf{0} & \mathbf{0} & \Phi_{x}{ }^{T} \Phi_{x}
\end{array}\right] d \xi
$$

with,

$$
\Delta=\left(l_{j} \xi\right)^{2}+2 l_{j} \xi \Phi_{x} \mathbf{q}_{\mathbf{e}_{\mathbf{x}_{\mathbf{j}}}}+\mathbf{q}_{\mathbf{e}_{\mathbf{x}_{\mathbf{j}}}}^{T} \Phi_{x}^{T} \Phi_{x} \mathbf{q}_{\mathbf{e}_{\mathbf{j}}}
$$

\section{Elastic Potential Energy}

The stiffness matrix, $\mathbf{K}_{\mathbf{j}}$, in Eqn. (4) is necessary for the evaluation of the elastic potential energy of a elastic beam. For beams in bending [?]:

$$
\mathbf{K}_{\mathbf{y}_{\mathbf{j}}}=\frac{E I_{j}}{l_{j}^{3}} \int_{0}^{1}\left(\frac{\partial^{2} u_{y}(\xi, t)}{\partial \xi^{2}}\right)^{2} d \xi
$$

Considering the first three modes of deformation, the stiffness matrix of a beam in pure bending is expressed as the diagonal matrix $\mathbf{K}_{\mathbf{y}}$ :

$$
\mathbf{K}_{\mathbf{y}_{\mathbf{j}}}=\frac{E I_{j}}{l_{j}^{3}} \int_{0}^{1}\left[\begin{array}{ccc}
\left(\frac{\partial^{2} \phi_{y_{1}}(\xi)}{\partial \xi^{2}}\right)^{2} & 0 & 0 \\
0 & \left(\frac{\partial^{2} \phi_{y_{2}}(\xi)}{\partial \xi^{2}}\right)^{2} & 0 \\
0 & 0 & \left(\frac{\partial^{2} \phi_{y_{3}}(\xi)}{\partial \xi^{2}}\right)^{2}
\end{array}\right] d \xi
$$


Similarly for beams in tension and compression:

$$
\mathbf{K}_{\mathbf{x}_{\mathbf{j}}}=\frac{E A_{j}}{l_{j}} \int_{0}^{1}\left(\frac{\partial u_{y}(\xi, t)}{\partial \xi}\right)^{2} d \xi
$$

Thus, for the first three modes:

$$
\mathbf{K}_{\mathbf{x}_{\mathbf{j}}}=\frac{E A_{j}}{l_{j}}\left[\begin{array}{ccc}
\int_{0}^{1}\left(\frac{\partial \phi_{x_{1}}(\xi)}{\partial \xi}\right)^{2} d \xi & 0 & 0 \\
0 & \int_{0}^{1}\left(\frac{\partial \phi_{x_{2}}(\xi)}{\partial \xi}\right)^{2} d \xi & 0 \\
0 & 0 & \int_{0}^{1}\left(\frac{\partial \phi_{x_{3}}(\xi)}{\partial \xi}\right)^{2} d \xi
\end{array}\right]
$$

Gravitational Potential Energy Finally the gravitational potential energy may be assessed. The gravitational potential energy $V_{g_{j}}$ for beam $j$ is determined at the beam mid-point using the mass $m_{j}$ of the link:

$$
V_{g_{j}}=\int_{m} \mathbf{g}^{T} \overrightarrow{O M^{*}}{ }_{j} d m=m_{j} \mathbf{g}^{T} \int_{0}^{1}{\overrightarrow{O M^{*}}}_{j} d \xi
$$

with $\overrightarrow{O M}_{j}$ :

$$
\overrightarrow{O M}_{j}=\underbrace{\left[\begin{array}{l}
x_{A_{j}} \\
y_{A_{j}}
\end{array}\right]+\mathbf{R}\left[\begin{array}{c}
\frac{l_{j}}{2} \\
0
\end{array}\right]}_{\overrightarrow{O M}_{j}}+\underbrace{\mathbf{R}\left[\begin{array}{l}
u_{x_{j}}(\xi, t) \\
u_{y_{j}}(\xi, t)
\end{array}\right]}_{\overrightarrow{M M}_{j}}
$$

with $u_{x_{j}}=\phi_{x_{j}}(\xi) \mathbf{q}_{\mathbf{e}_{\mathbf{j}}}, u_{y_{j}}=\phi_{y_{j}}(\xi) \mathbf{q}_{\mathbf{e}_{\mathbf{y}_{\mathbf{j}}}}$, and $\mathbf{g}=[0-g]^{T}$, the term for the gravitational effects can be found from:

$$
\mathbf{V}_{\mathbf{g}_{\mathbf{j}}}=\mathbf{m}_{\mathbf{j}} g\left(y_{A_{j}}+\frac{l}{2} \sin \left(\theta_{j}\right)+\alpha \mathbf{q}_{\mathbf{e}_{\mathbf{j}}}\right)
$$

where $\alpha$ is a vector depending on $\theta_{j}$.

\section{Equations of Motion}

The dynamics of the complete system with $n$ links may be solved by creating the total inertia and stiffness matrices, $\mathbf{M}_{t o t}$ and $\mathbf{K}_{\text {tot }}$. These matrices are block diagonal matrices formed by elements $\mathbf{M}_{1}$ to $\mathbf{M}_{n}$ and $\mathbf{K}_{\text {tot } 1}$ to $\mathbf{K}_{\text {totn } n}$ respectively. Note that:

$$
\mathbf{K}_{\mathbf{t o t}_{\mathbf{j}}}=\left[\begin{array}{cc}
\mathbf{0}_{3 \times 3} & \mathbf{0} \\
\mathbf{0} & \mathbf{K}_{\mathbf{j}}
\end{array}\right]
$$

The Lagrangian formulation of the elastodynamic problem for closed loop robots is developed from the Lagrangian defined in Eqn. (3).

$$
\mathbf{F}=\frac{d}{d t}\left[\frac{\partial L}{\partial \dot{\mathbf{q}}}\right]^{T}-\left[\frac{\partial L}{\partial \mathbf{q}}\right]+\Psi_{q}^{T} \lambda
$$

where,

F : is the vector sum of nonconservative external forces. In the absence of external forces this equals the joint torques, $\tau$

$\mathbf{q}=\left[\mathbf{q}_{\mathbf{1}}, \mathbf{q}_{\mathbf{2}}, \ldots, \mathbf{q}_{\mathbf{n}}\right]^{T}$, refers to the concatenation of the generalized coordinate vectors of each beam in the system, and,

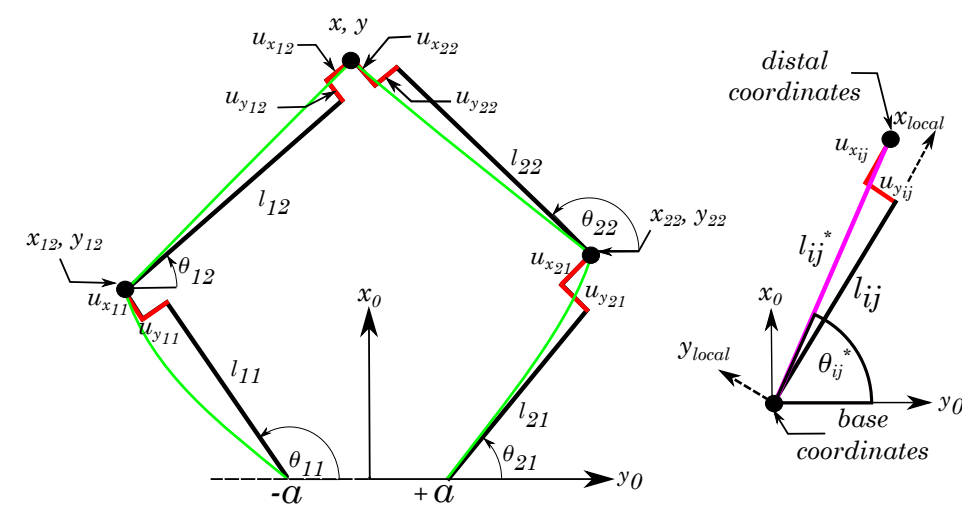

FIGURE 4. Elastic Geometric Model

$\mathbf{q}_{\mathbf{j}}=\left[x_{A_{j}}, y_{A_{j}}, \theta_{j}, \mathbf{q}_{\mathbf{e}_{\mathbf{j}}}\right]^{T}$

$\Psi_{q}=\frac{\partial \Psi}{\partial q}$ is $c \times N$ matrix with $c$ the number of constraints

and $N$ the total number of generalized coordinates. $\Psi=$ $\left[\begin{array}{lll}\mathbf{C}^{T} & \mathbf{B}^{T} & \mathbf{A}^{T}\end{array}\right]^{T}$, where the expressions of matrices $\mathbf{A}, \mathbf{B}$ and $\mathbf{C}$ are derived in the next section

$\lambda:$ is a $c \times 1$ vector of Lagrange multipliers

The $\Psi_{q}$ matrix is referred to as a Jacobian matrix in robotics literature and is responsible for the loop closure equations.

\section{Constraint Equations}

The constraint equations for the five bar mechanism are needed to solve for the terms of $\Psi_{q}$ : $\mathbf{A}, \mathbf{B}$ and $\mathbf{C}$. Referring to Fig. 4, the matrices correspond to the following groups:

1. A - passive coordinates

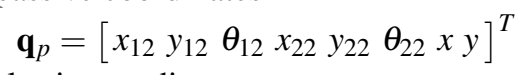

2. B - elastic coordinates

$$
\mathbf{q}_{e}=\left[\begin{array}{llll}
\mathbf{q}_{e_{11}} & \mathbf{q}_{e_{12}} & \mathbf{q}_{e_{21}} & \mathbf{q}_{e_{22}}
\end{array}\right]^{T}
$$

3. C - actuated coordinates, with $q_{11}=\theta_{11}$ and $q_{21}=\theta_{21}$

$$
\mathbf{q}_{a}=\left[\begin{array}{ll}
q_{11} & q_{21}
\end{array}\right]^{T}
$$

The coordinates $\left[x_{i j}, y_{i j}\right]$ refer to the base coordinates of beam $j$ in branch $i, \theta_{i j}$ refers to the orientation of the beam within the global frame. The vector $\mathbf{q}_{\mathbf{e}_{\mathrm{ij}}}$ refers to the set of deflection coordinates at the distal end of the beam. For the mechanism under consideration, the following constraint equations should be taken into account (Fig. 4):

$$
\begin{gathered}
{\left[\begin{array}{l}
f_{1} \\
f_{2}
\end{array}\right]=\mathbf{0}=\left[\begin{array}{l}
x \\
y
\end{array}\right]-\left[\begin{array}{l}
x_{12} \\
y_{12}
\end{array}\right]-l_{12}\left[\begin{array}{l}
\cos \theta_{12} \\
\sin \theta_{12}
\end{array}\right]-\mathbf{R}\left(\theta_{12}\right)\left[\begin{array}{l}
u_{x_{12}} \\
u_{y_{12}}
\end{array}\right]} \\
{\left[\begin{array}{l}
f_{3} \\
f_{4}
\end{array}\right]=\mathbf{0}=\left[\begin{array}{l}
x \\
y
\end{array}\right]-\left[\begin{array}{l}
x_{22} \\
y_{22}
\end{array}\right]-l_{22}\left[\begin{array}{l}
\cos \theta_{22} \\
\sin \theta_{22}
\end{array}\right]-\mathbf{R}\left(\theta_{22}\right)\left[\begin{array}{l}
u_{x_{22}} \\
u_{y_{22}}
\end{array}\right]} \\
{\left[\begin{array}{l}
f_{5} \\
f_{6}
\end{array}\right]=\mathbf{0}=\left[\begin{array}{l}
x_{12} \\
y_{12}
\end{array}\right]-\left[\begin{array}{c}
-a \\
0
\end{array}\right]-l_{11}\left[\begin{array}{l}
\cos q_{11} \\
\sin q_{11}
\end{array}\right]-\mathbf{R}\left(q_{11}\right)\left[\begin{array}{l}
u_{x_{11}} \\
u_{y_{11}}
\end{array}\right]} \\
{\left[\begin{array}{l}
f_{7} \\
f_{8}
\end{array}\right]=\mathbf{0}=\left[\begin{array}{l}
x_{22} \\
y_{22}
\end{array}\right]-\quad\left[\begin{array}{l}
a \\
0
\end{array}\right]-l_{21}\left[\begin{array}{l}
\cos q_{21} \\
\sin q_{21}
\end{array}\right]-\mathbf{R}\left(q_{21}\right)\left[\begin{array}{l}
u_{x_{21}} \\
u_{y_{21}}
\end{array}\right]}
\end{gathered}
$$


the rotation matrices being defined as follows:

$$
\mathbf{R}(\theta)=\left[\begin{array}{cc}
\cos \theta & -\sin \theta \\
\sin \theta & \cos \theta
\end{array}\right]
$$

For assumed modes, the deformations $u_{x}$ and $u_{y}$ are expressed in terms of the shape functions and elastic deformations:

$$
u_{x}=\Phi_{x}(\xi) \mathbf{q}_{\mathbf{e x}}(t) \quad u_{y}=\Phi_{y}(\xi) \mathbf{q}_{\mathbf{e y}}(t)
$$

Matrices A, $\mathbf{B}$ and $\mathbf{C}$ can thus be derived as:

$$
\mathbf{A}=\frac{\partial \mathbf{f}}{\partial \mathbf{q}_{\mathbf{p}}}, \mathbf{B}=\frac{\partial \mathbf{f}}{\partial \mathbf{q}_{\mathbf{e}}}, \mathbf{C}=\frac{\partial \mathbf{f}}{\partial \mathbf{q}_{\mathbf{a}}}
$$

with $\mathbf{f}=\left[f_{1}, f_{2}, \ldots, f_{8}\right]^{T}$.

\section{Direct and Inverse Dynamic Models}

The preceding equations may be used to develop the direct and inverse dynamic models of the closed loop robot. In the direct dynamic model, the generalized efforts $\tau$ are known and the independent coordinate accelerations $\ddot{\mathbf{q}}_{\mathbf{i}}=\left[\ddot{\mathbf{q}}_{\mathbf{a}}, \ddot{\mathbf{q}}_{\mathbf{e}}\right]$ are sought. In the inverse dynamic problem, $\ddot{\mathbf{q}}_{\mathbf{a}}$ is known and the required efforts $\tau$ and emergent $\ddot{\mathbf{q}}_{\mathbf{e}}$ are sought.

\section{TRAJECTORY DESIGN Geometric Path}

In this section geometric paths and motion profiles from which various trajectories may be generated are introduced. The following paths are used to smoothen the corners of the Adept cycle (see Fig. 5):

1. Clothoids; These curves are the time optimal solution for given constraints in jerk [?], [?]

2. Polar polynomials; they are expressed with closed form equations and represent the simplest blending approach [?]

3. Lamé Curves; contrary to the other types of curves, they allow a parametrization in Cartesian coordinates and are analytic along the required path being limited to the first quadrant [?] (in the remainder of the paper, Lamé Curves or order 3 are used)

An overlay of the three geometric paths is shown in Fig. 6, table 2 shows the lengths of the generated curves for $d=e=$ $0.25 \mathrm{~m}$. It can be seen that the Lamé curve generates the longest blending length while the polar polynomial generates the shortest one.

A motion profile is a monotonic function in $s$ describing the movement of the end effector along the geometric path:

$$
X(t)=s(t)\left(X_{f}-X_{i}\right)+X_{i}
$$

where

$X$ represents the position at time $t$

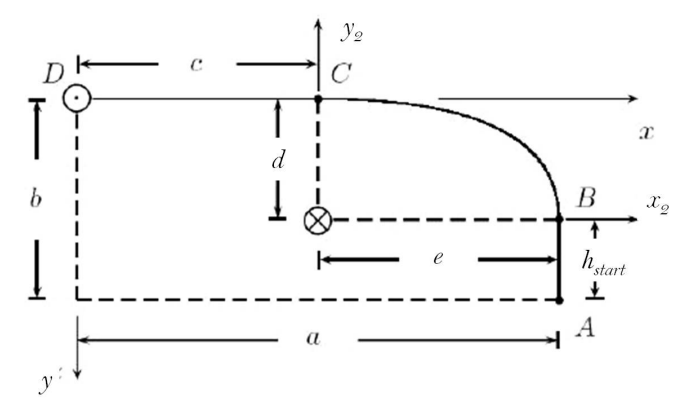

FIGURE 5. Adept half cycle using a Lamé curve [?]

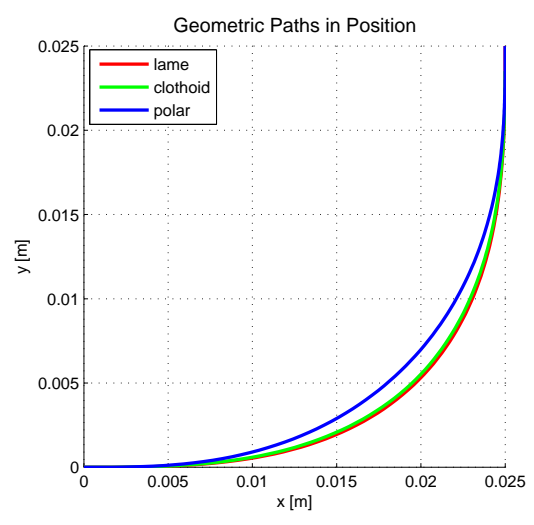

FIGURE 6. Blending sections for the adept cycle

$X_{f}$ is the final position, and,

$X_{i}$ is the initial position

A trajectory should be defined such that its acceleration profile is continuous. Pragmatism in industry however adopts profiles with discontinuities as they provide faster cycle times.

The motion profiles are designed to vary between $s=0$ and $s=1$, as a function of the normalized time $\eta=\frac{t}{t_{f}}$ ( $t_{f}$ represents the cycle time). The motion profiles are designed with respect to this cycle time $t_{f}$, the basis from which the necessary velocities and accelerations of the particular motion profile are found. These profile dictate the rate at which the end-effector follows the geometric path. In the remainder of this paper, the following profiles are investigated [?] (see Fig. 7):

1. Seventh degree polynomial profiles

2. Trapezoidal velocity profiles

3. Bang-bang acceleration profiles

\section{CASE STUDIES}

\section{Five Bar Mechanism Model}

The parameters of the five bar mechanism under study are given in Table 3. The assumed modes model was compared to 
TABLE 2. Blending lengths for geometric paths

\begin{tabular}{|c|c||c|c|}
\hline Curve Type & Length $[\mathrm{mm}]$ & Curve Type & Length $[\mathrm{mm}]$ \\
\hline \hline Lamé & 42.156 & Extended Lamé & 59.307 \\
Clothoid & 41.333 & Extended Clothoid & 57.015 \\
Polar & 41.106 & & \\
\hline
\end{tabular}

TABLE 3. Parameters of the modeled five bar mechanism

\begin{tabular}{|c|c|c|}
\hline Links & $l_{i j}$ Length $[\mathrm{m}]$ & $D_{i j}$ Diameter $[d]$ \\
\hline \hline 11,21 & 0.24 & 0.035 \\
12,22 & 0.38079 & 0.035 \\
\hline \hline \multicolumn{2}{|c|}{ Other Parameters } \\
\hline \hline Material & \multicolumn{2}{|c|}{ Aluminium } \\
\hline$\rho$ & Density $\left[\mathrm{kg} / \mathrm{m}^{3}\right]$ & 2710 \\
$Y$ & Young's Modulus $[\mathrm{GPa}]$ & 70 \\
\hline
\end{tabular}
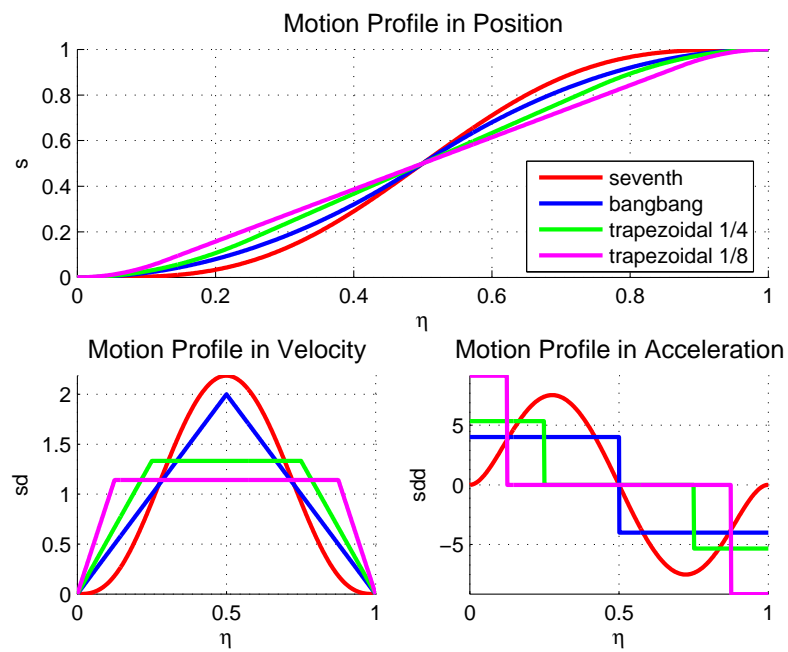

Motion Profile in Acceleration

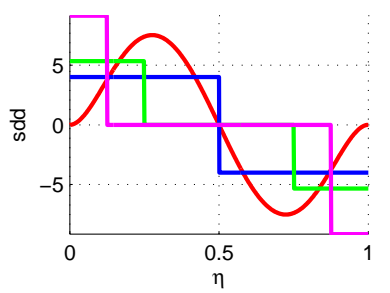

FIGURE 7. Motion profiles generated for $t_{f}=1$

a finite element model built in Castem [?]. Confidence in the model was ascertained by validating the natural frequencies and loaded deformations of the system.

\section{Trajectory Characteristics}

In this section, the effect of transversing a particular geometric path with a given motion profile is studied.

The profiles and paths listed in Table 4 allow 12 different path-profile combinations to be analyzed. At this point, the motion profiles are generated for a cycle time of 1 second, various characteristics of the generated trajectories may then be investigated. The $\frac{1}{4}$ and $\frac{1}{8}$ trapezoidal profiles refer to acceleration
TABLE 4. Tested motion profiles and geometric paths

\begin{tabular}{|c|c|}
\hline Motion Profiles & Geometric Paths \\
\hline $7^{\text {th }}$ order polynomial & Lamé curve \\
Bang-bang & Clothoid pair \\
$\frac{1}{4}$ Trapezoidal & Polar polynomial \\
$\frac{1}{8}$ Trapezoidal & \\
\hline
\end{tabular}

TABLE 5. Task space acceleration comparison

\begin{tabular}{|c||c|c|c|}
\hline \multicolumn{1}{|c||}{} & \multicolumn{3}{c|}{ Acceleration $\left[\mathrm{m} / \mathrm{s}^{2}\right]$} \\
\hline Motion Profiles & Lamé & Clothoid & Polar \\
\hline \hline $7^{\text {th }}$ order polynomial & 7.7818 & 7.3686 & 7.0384 \\
Bang-bang & 5.1687 & 4.7268 & 4.4662 \\
$\frac{1}{4}$ Trapezoidal & 6.8895 & 6.2916 & 5.9544 \\
$\frac{1}{8}$ Trapezoidal & 11.8115 & 10.7638 & 10.0453 \\
\hline
\end{tabular}

durations of $\frac{t_{f}}{4}$ and $\frac{t_{f}}{8}$ respectively.

Task Space Table 5 shows a comparison of the acceleration characteristics of each desired trajectory in the task space. A trend that can be observed is that the Lamé curve requires higher accelerations because it has the longest curve length (Table 2).

Joint Space The effect of trajectory following on the five bar mechanism in the joint space is now investigated. The joint trajectories are computed with the inverse geometric model and are assessed with respect to:

1. motion profile - geometric path combinations

2. cycle times

3. blending lengths

Figure 8 shows the articulated joint displacements for the trajectory plotted in Fig. 9 and for $t_{f}=1$. The spectral content of each signal in acceleration is determined. The figure shows the Power Spectral Density (PSD) function of each signal. This distribution of energy is important as it gives an indication to the extent whereby the resonance frequencies of the structure will be excited by each trajectory. It can be observed that the spectral content of the $7^{\text {th }}$ degree polynomial is much more forgiving than those of the trapezoidal and bang-bang profiles.

The features of each trajectory in the task space are shown in Table 6. Figures 10 and 11 show the effect of varying the cycle time and the blending length $d$. As expected, decreasing the cycle time $t_{f}$ inflates the energy content at all frequencies. Furthermore, the shorter the blending length, and thus, the sharper the corner, the higher the energy content of the input signal.

Trajectory Discussion It is clear that there is a tradeoff between the cycle time and the amount of energy distribu- 

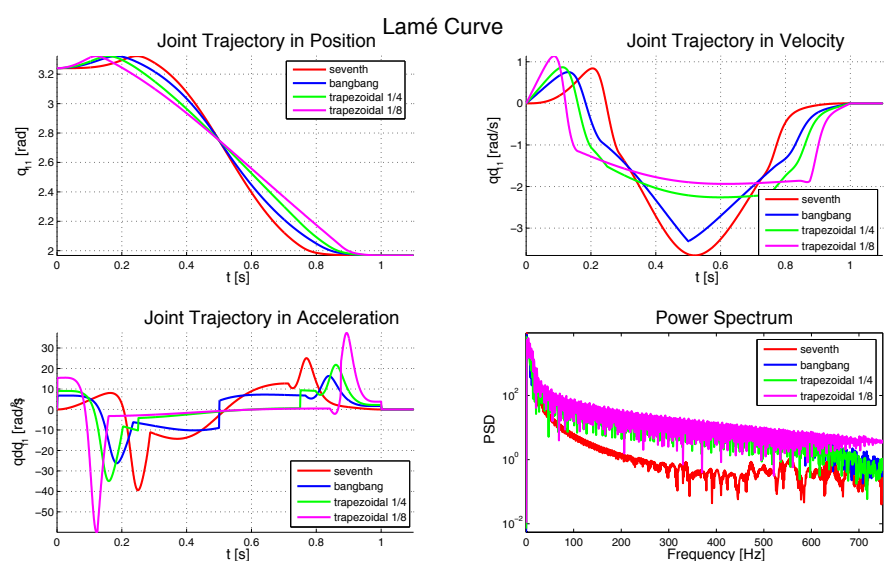

FIGURE 8. Lame curve joint space trajectory and spectral content

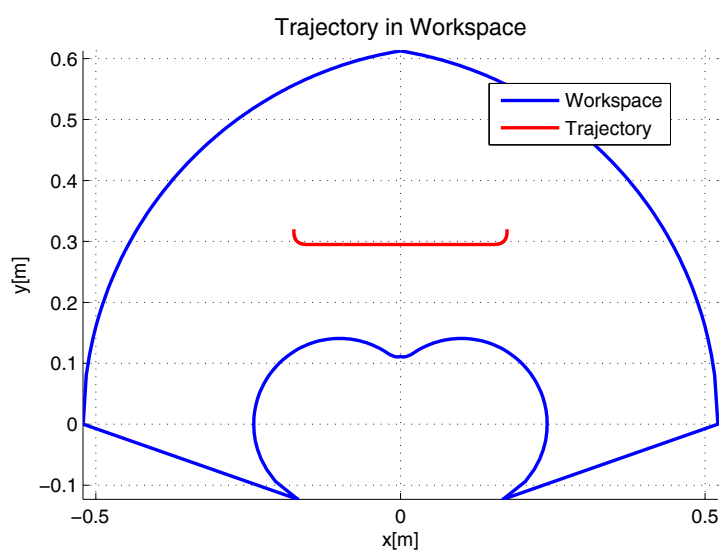

FIGURE 9. Trajectory placement in workspace

TABLE 6. Joint space acceleration comparison

\begin{tabular}{|c||c|c|c|}
\hline \multicolumn{1}{|c||}{} & \multicolumn{3}{|c|}{ Acceleration $\left[\mathrm{rad} / \mathrm{s}^{2}\right]$} \\
\hline Motion Profiles & Lamé & Clothoid & Polar \\
\hline \hline $7^{\text {th }}$ order polynomial & 25.0858 & 25.3279 & 21.2023 \\
Bang-bang & 16.4343 & 16.4834 & 13.7179 \\
$\frac{1}{4}$ Trapezoidal & 21.8879 & 21.9759 & 18.1215 \\
$\frac{1}{8}$ Trapezoidal & 37.432 & 37.6681 & 30.9457 \\
\hline
\end{tabular}

tion in the trajectory signals. The following conclusions may be drawn:

1. Shorter curve lengths require lower accelerations

2. Lower cycle times require higher accelerations and increase the spectral content of the signal at all frequencies

3. Sharp corners increase the spectral content of the signal at
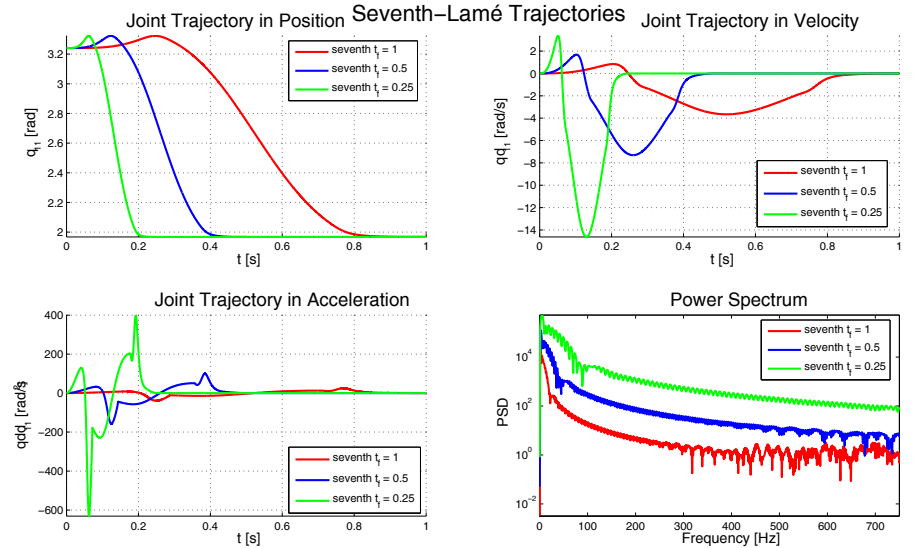

FIGURE 10. Spectral content for seventh-lamé trajectories for cycle times of $t_{f}=1, t_{f}=0.5$ and $t_{f}=0.25$
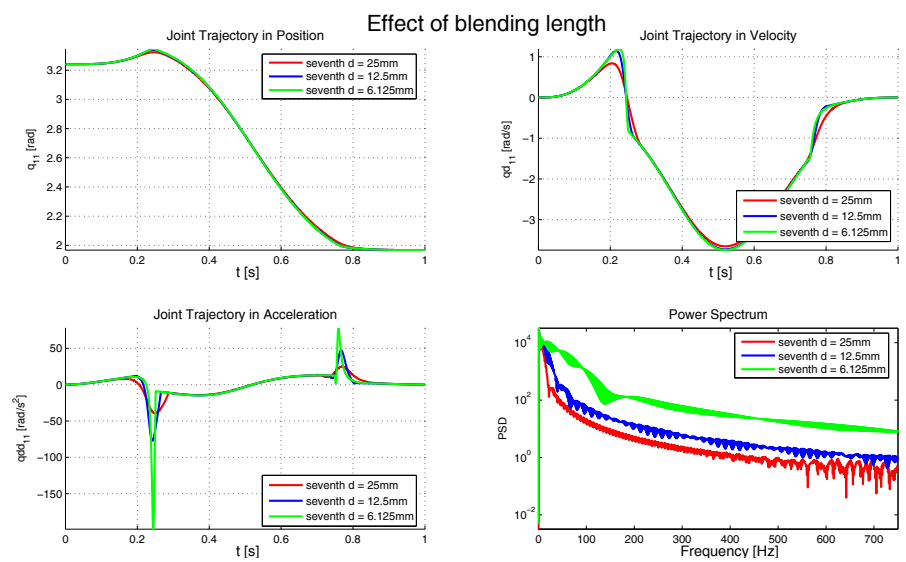

FIGURE 11. Spectral content for seventh-lamé trajectories for symmetric blending lengths of $d=25 \mathrm{~mm}, d=12.5 \mathrm{~mm}$ and $d=6.125 \mathrm{~mm}$

all frequencies

In the upcoming section the elastodynamic model of the five bar mechanism will be excited with the trajectories discussed in this section.

It is assumed that a perfect controller is available. The inverse elastodynamic model is used to evaluate the vibratory performance of the five bar mechanism as it traverses the various trajectories. The simulations are conducted for a payload of $0.1 \mathrm{~kg}$ and $6.0 \mathrm{~kg}$. The simulation is run at a sampling frequency of $10 \mathrm{MHz}$. Note that gravity is taken upwards.

The following results will be presented and discussed:

1. Trajectory following in the task space

2. Required joint torques

3. Excited vibrations 
TABLE 7. Performances comparison along the generated trajectories

\begin{tabular}{|c||c|c|c|}
\hline \multicolumn{1}{|c||}{} & Lamé & Clothoid & Polar \\
\hline \hline Motion Profiles & \multicolumn{3}{|c|}{ Torque $[\mathrm{Nm}]$} \\
\hline $7^{\text {th }}$ order polynomial & 73.634 & 70.046 & 71.298 \\
Bang-bang & 56.084 & 50.848 & 50.734 \\
$\frac{1}{4}$ Trapezoidal & 75.208 & 74.605 & 62.496 \\
$\frac{1}{8}$ Trapezoidal & 125.45 & 124.45 & 119.16 \\
\hline Motion Profiles & RMS Acceleration Error $\left[\mathrm{m} / \mathrm{s}^{2}\right]$ \\
\hline $7^{\text {th }}$ order polynomial & 2.7168 & 3.3724 & 6.641 \\
Bang-bang & 4.5245 & 4.7786 & 5.4098 \\
$\frac{1}{4}$ Trapezoidal & 6.0193 & 6.3989 & 8.6868 \\
$\frac{1}{8}$ Trapezoidal & 10.572 & 10.731 & 13.865 \\
\hline Motion Profiles & \multicolumn{3}{|c|}{ Max Deflection $[\mathrm{mm}]$} \\
\hline $7^{\text {th }}$ order polynomial & 0.09700 & 0.09226 & 0.09392 \\
Bang-bang & 0.07387 & 0.06696 & 0.06681 \\
$\frac{1}{4}$ Trapezoidal & 0.09911 & 0.09832 & 0.08233 \\
$\frac{1}{8}$ Trapezoidal & 0.16540 & 0.16408 & 0.13813 \\
\hline
\end{tabular}

4. Spectral content of the vibratory signal

\section{Simulation Set 1: $6.0 \mathrm{~kg}, t_{f}=0.43 \mathrm{~s}$ Vibrational Domain}

With a payload of $6 \mathrm{~kg}$, a frequency analysis along the geometric curve reveals that the first natural frequency of the robot varies with $\pm 1 \mathrm{~Hz}$ about $58 \mathrm{~Hz}$.

From Table 7, it can be seen that high input accelerations, and thus high torques, correspond to the highest deflections, while the opposite is true for low input accelerations. From the investigation, it can be seen that the vibratory content of a given signal depends on the smoothness of the trajectory, with less oscillations attributable to the $7^{\text {th }}$ degree polynomial. The smoother the trajectory and geometric path, the less oscillatory the signal, this is revealed through the RMS error values.

Errors in the Task Space The norm of the error in the task space for a payload of $6 \mathrm{~kg}$ and a cycle time of $t_{f}=0.43 \mathrm{~s}$ is shown in Fig. 12. The results are detailed in Table 8. Although the bang-bang profile yields a lower residual error norm in position at $t_{f}=0.43 \mathrm{~s}$ than the $7^{t} h$ order polynomial, it can be seen that the residual errors in velocity and acceleration are attenuated to greater extent by the $7^{\text {th }}$ order polynomial. Therefore, bangbang trajectories lead to poorer vibration attenuation.
TABLE 8. Residual error norms at end effector end points for a payload of $6 \mathrm{~kg}$

\begin{tabular}{|c||c|c|c|}
\hline \multicolumn{1}{|c||}{} & Lamé & Clothoid & Polar \\
\hline \hline Motion Profiles & \multicolumn{3}{c|}{ Position $[\mathrm{mm}]$} \\
\hline $7^{\text {th }}$ order polynomial & 0.9355 & 0.0009 & 0.3109 \\
Bang-bang & 4.8557 & 0.0007 & 0.5573 \\
$\frac{1}{4}$ Trapezoidal & 5.5411 & 0.0017 & 0.9366 \\
$\frac{1}{8}$ Trapezoidal & 8.7118 & 0.0049 & 0.2872 \\
\hline Motion Profiles & \multicolumn{3}{|c|}{ Velocity $[\mathrm{m} / \mathrm{s}]$} \\
\hline $7^{\text {th }}$ order polynomial & 0.0009 & 0.0053 & 0.4973 \\
Bang-bang & 0.0049 & 0.0050 & 1.0716 \\
$\frac{1}{4}$ Trapezoidal & 0.0055 & 0.0055 & 0.2611 \\
$\frac{1}{8}$ Trapezoidal & 0.0087 & 0.0062 & 0.8352 \\
\hline Motion Profiles & \multicolumn{3}{|c|}{ Acceleration $\left[\mathrm{m} / \mathrm{s}^{2}\right]$} \\
\hline $7^{\text {th }}$ order polynomial & 0.5573 & 0.0045 & 1.3193 \\
Bang-bang & 0.4973 & 0.0087 & 3.8472 \\
$\frac{1}{4}$ Trapezoidal & 0.8352 & 0.0056 & 1.8212 \\
$\frac{1}{8}$ Trapezoidal & 1.8212 & 0.0083 & 3.0404 \\
\hline
\end{tabular}
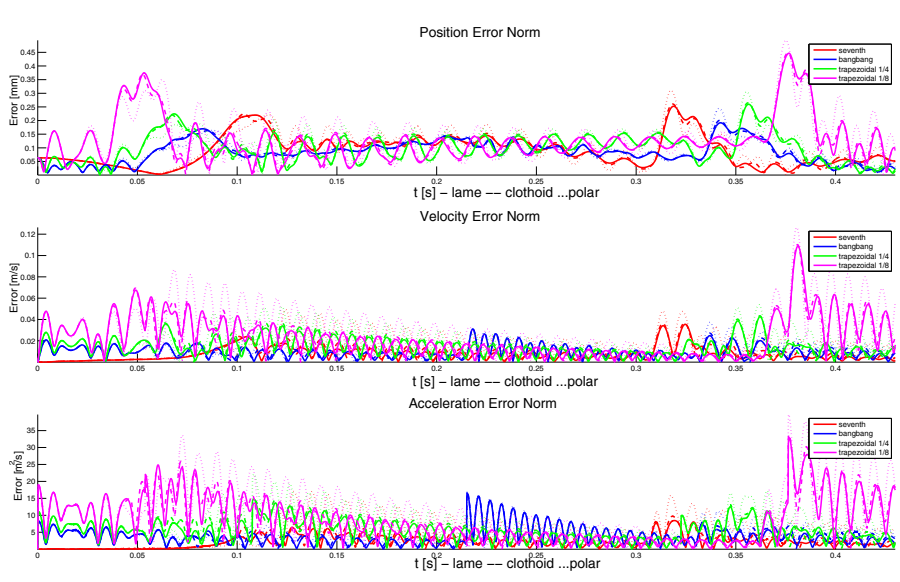

FIGURE 12. Path tracking errors in the task space for the payload of $6 \mathrm{~kg}$.

\section{Simulation Set 2: Payload of $0.1 \mathrm{~kg}, t_{f}=0.3 \mathrm{~s}$}

\section{Vibrational Domain}

With a payload of $0.1 \mathrm{~kg}$, a frequency analysis along the geometric curve reveals that the first natural frequency of the robot varies with $\pm 2 \mathrm{~Hz}$ about $126 \mathrm{~Hz}$, while the margin increases to $\pm 7.5 \mathrm{~Hz}$ about $1912 \mathrm{~Hz}$ for the third natural frequency. 
TABLE 9. Task space comparison along the generated trajectories

\begin{tabular}{|c||c|c|c|}
\hline \multicolumn{1}{|c||}{} & Lamé & Clothoid & Polar \\
\hline \hline Motion Profiles & \multicolumn{3}{|c|}{ Torque $[\mathrm{Nm}]$} \\
\hline $7^{\text {th }}$ order polynomial & 33.6384 & 32.737 & 33.0867 \\
Bang-bang & 23.9012 & 23.1011 & 22.3707 \\
$\frac{1}{4}$ Trapezoidal & 30.6662 & 29.4722 & 28.56 \\
$\frac{1}{8}$ Trapezoidal & 51.3054 & 45.9056 & 47.4447 \\
\hline Motion Profiles & RMS Acceleration Error $\left[\mathrm{m} / \mathrm{s}^{2}\right]$ \\
\hline $7^{\text {th }}$ order polynomial & 3.3563 & 3.8525 & 6.2169 \\
Bang-bang & 5.2095 & 5.3852 & 5.7002 \\
$\frac{1}{4}$ Trapezoidal & 5.2813 & 5.6053 & 5.9695 \\
$\frac{1}{8}$ Trapezoidal & 9.8592 & 8.7226 & 9.5968 \\
\hline Motion Profiles & Max End-effector Deflection $[\mathrm{mm}]$ \\
\hline $7^{\text {th }}$ order polynomial & 0.04339 & 0.04223 & 0.04269 \\
Bang-bang & 0.03082 & 0.02979 & 0.02885 \\
$\frac{1}{4}$ Trapezoidal & 0.03957 & 0.03802 & 0.03684 \\
$\frac{1}{8}$ Trapezoidal & 0.0663 & 0.05931 & 0.06134 \\
\hline
\end{tabular}

\section{Simulation Results}

The results of the simulation are summarized in Table 9. It can be observed that the end-effector states in position are the best for the bang-bang profile, as the bang-bang profile has the lowest acceleration value. Moreover, the $7^{\text {th }}$ degree polynomial offers the best performance in acceleration error, owing to the continuity and smoothness of its acceleration profile at this point.

From the table it can be seen that the worst performance, on all counts, is associated with the $\frac{1}{8}$ trapezoidal profile as it requires the highest accelerations. The required torque is related to the path length, and thus the lowest torques correspond to the polar polynomial trajectories. The best performance in terms of the RMS acceleration error is associated with the smoothest trajectory, i.e., the $7^{\text {th }}$ degree polynomial-Lamé curve.

Residual errors in the Task Space The norm of the error in the task space is summarized in Table 10. While the smoothness of a curve significantly decreases the residual error, the maximum acceleration achieved along a path plays a dominant role. The trends correspond to those seen earlier. The polar polynomial has slightly higher spectral energy at the natural frequencies of the robot and is associated with higher residual errors.

It is apparent that the smooth $7^{\text {th }}$ order curve presents the best results in the error norms. At this slower cycle speed of $t_{f}=$ 0.3 and low payload of $100 \mathrm{~g}$, the smoothness of the trajectory is
TABLE 10. Residual norms at end effector end points

\begin{tabular}{|c||c|c|c|}
\hline \multicolumn{1}{|c||}{} & Lamé & Clothoid & Polar \\
\hline \hline Motion Profiles & \multicolumn{3}{|c|}{ Position $[\mathrm{mm}]$} \\
\hline $7^{\text {th }}$ order polynomial & 0.0157 & 0.0009 & 0.3109 \\
Bang-bang & 0.0078 & 0.0007 & 0.5573 \\
$\frac{1}{4}$ Trapezoidal & 0.0160 & 0.0017 & 0.9366 \\
$\frac{1}{8}$ Trapezoidal & 0.0439 & 0.0049 & 0.2872 \\
\hline Motion Profiles & \multicolumn{3}{|c|}{ Velocity $[\mathrm{m} / \mathrm{s}]$} \\
\hline $7^{\text {th }}$ order polynomial & 0.0009 & 0.0053 & 0.4973 \\
Bang-bang & 0.0049 & 0.0050 & 1.0716 \\
$\frac{1}{4}$ Trapezoidal & 0.0055 & 0.0055 & 0.2611 \\
$\frac{1}{8}$ Trapezoidal & 0.0087 & 0.0062 & 0.8352 \\
\hline Motion Profiles & \multicolumn{3}{|c|}{ Acceleration $\left[\mathrm{m} / \mathrm{s}^{2}\right]$} \\
\hline $7^{\text {th }}$ order polynomial & 0.5573 & 0.0045 & 1.3193 \\
Bang-bang & 0.4973 & 0.0050 & 1.0716 \\
$\frac{1}{4}$ Trapezoidal & 0.8352 & 0.0045 & 1.3193 \\
$\frac{1}{8}$ Trapezoidal & 1.8212 & 0.0083 & 3.0404 \\
\hline
\end{tabular}

the dominant factor for the level of residual activity in position.

\section{Simulation Set 3: Extended Curve Lengths}

This section deals with the effect of varying the blending lengths of the clothoid pairs and the Lamé curve. The blending curves with $d=e=25 \mathrm{~mm}$ are compared with a Lamé curve with $e=125 \mathrm{~mm}$ and a clothoid pair with $e=44 \mathrm{~mm}$ (referred to as extended curves).

Table 11 shows the error norms in position, velocity and acceleration. It can be observed that the shortest trajectory, i.e., the extended Lamé curve, shows the best performance on all counts. In general it can be shown that shorter path lengths yield better results, with the $7^{\text {th }}$ order polynomial providing the best results in terms of velocity and acceleration.

\section{CONCLUSIONS}

This research investigated the definition of an optimal motion generator for a five-bar mechanism performing pick and place tasks. Several path and motion plannings have been simulated using an elastodynamic model of a flexible five-bar mechanism.

The simulation results showed the following:

1. The pick and place trajectories only excite the first and second natural frequencies of the five bar mechanism; 
TABLE 11. Comparison of error norms on short and extended cycle lengths

\begin{tabular}{|c||c|c|c|c|}
\hline \multicolumn{1}{|c||}{} & Lamé & Clot. & Lamé Ext & Clot. Ext \\
\hline \hline Motion Profiles & \multicolumn{4}{c|}{ Total Path Length $[\mathrm{mm}]$} \\
\hline \multicolumn{1}{|c||}{ N/A } & 0.3843 & 0.3840 & 0.3687 & 0.3751 \\
\hline Motion Profiles & \multicolumn{4}{|c|}{ Position $[\mathrm{mm}]$} \\
\hline $7^{\text {th }}$ order polynomial & 0.0524 & 0.0503 & 0.0606 & 0.0905 \\
Bang-bang & 0.0219 & 0.0293 & 0.0125 & 0.0461 \\
$\frac{1}{4}$ Trapezoidal & 0.0158 & 0.0221 & 0.0056 & 0.0320 \\
$\frac{1}{8}$ Trapezoidal & 0.0968 & 0.0919 & 0.0441 & 0.0659 \\
\hline Motion Profiles & \multicolumn{4}{|c|}{ Velocity $[\mathrm{m} / \mathrm{s}]$} \\
\hline $7^{\text {th }}$ order polynomial & 0.0014 & 0.0044 & 0.0005 & 0.0058 \\
Bang-bang & 0.0085 & 0.0102 & 0.0007 & 0.0039 \\
$\frac{1}{4}$ Trapezoidal & 0.0122 & 0.0130 & 0.0134 & 0.0138 \\
$\frac{1}{8}$ Trapezoidal & 0.0162 & 0.0253 & 0.0081 & 0.0283 \\
\hline Motion Profiles & \multicolumn{4}{|c||}{ Acceleration $\left[\mathrm{m} / \mathrm{s}^{2}\right]$} \\
\hline $7^{\text {th }}$ order polynomial & 1.7121 & 2.0211 & 0.3816 & 0.2521 \\
Bang-bang & 1.6179 & 2.9177 & 0.2230 & 0.8533 \\
$\frac{1}{4}$ Trapezoidal & 1.6563 & 2.2635 & 1.0501 & 1.2532 \\
$\frac{1}{8}$ Trapezoidal & 18.1496 & 17.4328 & 8.9629 & 12.2502 \\
\hline
\end{tabular}

2. The maximum deflection along the path is directly related to the maximum torque required to complete the given trajectory in a given cycle time $t_{f}$;

3. The shorter the path length, the lower the required acceleration and as a result the lower the required torques;

4. The terminal state accuracy can be attributed to the smoothness of the trajectory, with $7^{\text {th }}$ degree polynomial-Lamé trajectories showing the best results.

For the future works, it is envisioned that trajectory optimization may take place as well as the inclusion of conventional feedback controllers coupled with input shapers for the reduction of residual vibrations.

\section{ACKNOWLEDGMENTS}

This work has been partially funded by the French ANR project ARROW (ANR 2011 BS3 006 01). 